\title{
Kernel Theorems in Spaces of Tempered Generalized Functions
}

\author{
A. Delcroix \\ Equipe Analyse Algébrique Non Linéaire \\ Laboratoire Analyse, Optimisation, Contrôle \\ Faculté des sciences - Université des Antilles et de la Guyane \\ 97159 Pointe-à-Pitre Cedex \\ Guadeloupe \\ E_mail: Antoine.Delcroix@univ-ag.fr
}

September 23, 2018

\begin{abstract}
In analogy to the classical isomorphism between $\mathcal{L}\left(\mathcal{S}\left(\mathbb{R}^{n}\right), \mathcal{S}^{\prime}\left(\mathbb{R}^{m}\right)\right)$ and $\mathcal{S}^{\prime}\left(\mathbb{R}^{n+m}\right)$, we show that a large class of moderate linear mappings acting between the space $\mathcal{G}_{\mathcal{S}}\left(\mathbb{R}^{n}\right)$ of Colombeau rapidly decreasing generalized functions and the space $\mathcal{G}_{\tau}\left(\mathbb{R}^{n}\right)$ of temperate ones admits generalized integral representations, with kernels belonging to $\mathcal{G}_{\tau}\left(\mathbb{R}^{n+m}\right)$. Furthermore, this result contains the classical one in the sense of the generalized distribution equality.
\end{abstract}

Mathematics Subject Classification (2000): 45P05, 46F05, 46F30, 47G10

Keywords: kernel Theorem, Colombeau temperate generalized functions, integral operator, temperate distributions.

\section{Introduction}

During the three last decades, theories of nonlinear generalized functions have been developed by many authors (see 11, 11, 14, 15, ,..), mainly based on the ideas of J.-F. Colombeau [3, 4], which we are going to follow in the sequel. Those theories appear to be a natural continuation of the distributions' one [12, 21, 22, specially efficient to pose and solve differential or integral problems with irregular data.

In this paper, we continue the investigations in the field of generalized integral operators initiated by [18, (recently republished in [19, 20]) and carried on by [2, 5, 9, 10, 23. Let us recall that those operators generalize, in the Colombeau framework, the operators with distributional kernels in the space of Schwartz distributions [2]. More specifically, in [5], we proved that any moderate net of linear maps $\left(L_{\varepsilon}: \mathcal{D}\left(\mathbb{R}^{n}\right) \rightarrow \mathrm{C}^{\infty}\left(\mathbb{R}^{m}\right)\right)_{\varepsilon}$, that is satisfying some growth properties with respect to the parameter $\varepsilon$, gives rise to a linear map $L: \mathcal{G}_{C}\left(\mathbb{R}^{n}\right) \rightarrow \mathcal{G}\left(\mathbb{R}^{m}\right)$. (Where $\mathcal{G}\left(\mathbb{R}^{d}\right)$ and $\mathcal{G}_{C}\left(\mathbb{R}^{n}\right)$ denote respectively the space of generalized functions and the space of compactly supported ones.) The main result is that $L$ can be represented as a generalized integral operator in the spirit of Schwartz Kernel Theorem.

Going further in this direction, we study here the generalization of the classical isomorphism between $\mathcal{S}^{\prime}\left(\mathbb{R}^{n+m}\right)$ and the space of continuous linear mappings acting between $\mathcal{S}\left(\mathbb{R}^{n}\right)$ and $\mathcal{S}^{\prime}\left(\mathbb{R}^{m}\right)$ 22. Thus, the spaces of generalized functions considered in this paper are the space $\mathcal{G}_{\mathcal{S}}\left(\mathbb{R}^{n}\right)$ of rapidly decreasing generalized functions [7, 10, 17] and the space $\mathcal{G}_{\tau}\left(\mathbb{R}^{n}\right)$ of tempered generalized functions 4, 11, 18: $\mathcal{G}_{\mathcal{S}}\left(\mathbb{R}^{n}\right)$ plays here, roughly speaking, the role of $\mathcal{S}\left(\mathbb{R}^{n}\right)\left(\right.$ resp. $\left.\mathcal{G}_{C}\left(\mathbb{R}^{n}\right)\right)$ in the classical case (resp. in [5]), whereas $\mathcal{G}_{\tau}\left(\mathbb{R}^{n}\right)$ plays the role of $\mathcal{S}^{\prime}\left(\mathbb{R}^{m}\right)$ (resp. $\mathcal{G}\left(\mathbb{R}^{m}\right)$ ) in the classical case (resp. in [5]). 
The main results are the following. First, any kernel $H \in \mathcal{G}_{\tau}\left(\mathbb{R}^{m+n}\right)$ gives rise to a new type of linear generalized integral operator acting between $\mathcal{G}_{\mathcal{S}}\left(\mathbb{R}^{n}\right)$ and $\mathcal{G}_{\tau}\left(\mathbb{R}^{m}\right)$ and defined by

$$
\widetilde{H}: \quad \mathcal{G}_{\mathcal{S}}\left(\mathbb{R}^{n}\right) \rightarrow \mathcal{G}_{\tau}\left(\mathbb{R}^{m}\right), \quad f \mapsto \widetilde{H}(f)=\left[\left(x \mapsto \int H_{\varepsilon}(x, y) f_{\varepsilon}(y) \mathrm{d} y\right)_{\varepsilon}\right]_{\tau},
$$

where $\left(H_{\varepsilon}\right)_{\varepsilon}\left(\right.$ resp. $\left.\left(f_{\varepsilon}\right)_{\varepsilon}\right)$ is any representative of $H$ (resp. $f$ ) and $[\cdot]_{\tau}$, the class of an element in $\mathcal{G}_{\tau}\left(\mathbb{R}^{m}\right)$. Moreover, the linear map $H \mapsto \widetilde{H}$ from $\mathcal{G}_{\tau}\left(\mathbb{R}^{m+n}\right)$ to the space of linear maps $\mathbf{L}\left(\mathcal{G}_{\mathcal{S}}\left(\mathbb{R}^{n}\right), \mathcal{G}_{\tau}\left(\mathbb{R}^{m}\right)\right.$ ) is injective (Proposition 14). This gives the first part of the expected result. Then, new regular subspaces of $\mathcal{G}_{\mathcal{S}}\left(\mathbb{R}^{n}\right)$ and $\mathcal{G}_{\tau}\left(\mathbb{R}^{m}\right)$ are introduced in the spirit of [6]. They are used to define the moderate nets of linear maps, which can be extended to act between $\mathcal{G}_{\mathcal{S}}\left(\mathbb{R}^{n}\right)$ and $\mathcal{G}_{\tau}\left(\mathbb{R}^{m}\right)$ (Proposition 16). Finally, our main result states that those extensions can be represented as generalized integral operators (Theorem 17). Furthermore, this result is strongly related to the classical isomorphism theorem recalled above in the following sense. We can associate to each linear continuous operator $\Lambda: \mathcal{S}\left(\mathbb{R}^{n}\right) \rightarrow \mathcal{S}^{\prime}\left(\mathbb{R}^{m}\right)$, a moderate map $L_{\Lambda}$ and consequently a kernel $H_{L_{\Lambda}} \in \mathcal{G}_{\tau}\left(\mathbb{R}^{m+n}\right)$ such that, for all $f$ in $\mathcal{S}\left(\mathbb{R}^{n}\right), \Lambda(f)$ and $\widetilde{H}_{L_{\Lambda}}(f)$ are equal in the generalized distribution sense [14] (Proposition [20).

The paper can be divided in two parts. The first part, formed by section 2 and section 3 introduces the material which is needed in the sequel (regular spaces of generalized functions, generalized integral operators). The second part, consisting in the two last sections, is devoted to the definition of moderate nets, the statement of the main theorems and their proofs. Concerning them, we insist on the differences with [5]: Replacing $\mathcal{G}_{C}\left(\mathbb{R}^{n}\right)$ by the bigger space $\mathcal{G}_{\mathcal{S}}\left(\mathbb{R}^{n}\right)$ and $\mathcal{G}\left(\mathbb{R}^{m}\right)$ by $\mathcal{G}_{\tau}\left(\mathbb{R}^{m}\right)$ obliges to consider global estimates instead of ones on compact sets. This forces to introduce a new concept of moderate maps, and to refine the estimates and the arguments concerning integration. However, we also substantially simplify here the proof of the equality in generalized distribution sense.

\section{Colombeau type algebras}

Throughout this section, $d$ will be a strictly positive integer and $\Omega$ an open subset of $\mathbb{R}^{d}$. As mentioned in the introduction, we only consider in this paper the spaces $\mathcal{G}_{\mathcal{S}}(\Omega)$ of rapidly decreasing generalized functions and $\mathcal{G}_{\tau}(\Omega)$ of temperate generalized functions. For $f \in \mathrm{C}^{\infty}(\Omega), r \in \mathbb{Z}$ and $l \in \mathbb{N}$, set

$$
\left.\mu_{r, l}(f)=\sup _{x \in \Omega,|\alpha| \leq l}(1+|x|)^{r}\left|\partial^{\alpha} f(x)\right| \quad \text { (with values in }[0,+\infty]\right)
$$

\subsection{Rapidly decreasing generalized functions}

Set

$$
\begin{aligned}
\mathcal{E}_{\mathcal{S}}(\Omega) & =\left\{\left(f_{\varepsilon}\right)_{\varepsilon} \in \mathcal{S}(\Omega)^{(0,1]} \mid \forall(q, l) \in \mathbb{N}^{2}, \exists N \in \mathbb{N}, \quad \mu_{q, l}\left(f_{\varepsilon}\right)=\mathrm{O}\left(\varepsilon^{-N}\right) \text { as } \varepsilon \rightarrow 0\right\} \\
\mathcal{N}_{\mathcal{S}}(\Omega) & =\left\{\left(f_{\varepsilon}\right)_{\varepsilon} \in \mathcal{S}(\Omega)^{(0,1]} \mid \forall(q, l) \in \mathbb{N}^{2}, \forall p \in \mathbb{N}, \quad \mu_{q, l}\left(f_{\varepsilon}\right)=\mathrm{O}\left(\varepsilon^{p}\right) \text { as } \varepsilon \rightarrow 0\right\} .
\end{aligned}
$$

One can show that $\mathcal{E}_{\mathcal{S}}(\Omega)$ is a subalgebra of $\mathcal{S}(\Omega)^{(0,1]}$ and $\mathcal{N}_{\mathcal{S}}(\Omega)$ an ideal of $\mathcal{E}_{\mathcal{S}}(\Omega)$. The algebra $\mathcal{G}_{\mathcal{S}}(\Omega)=\mathcal{E}_{\mathcal{S}}(\Omega) / \mathcal{N}_{\mathcal{S}}(\Omega)$ is called the algebra of rapidly decreasing generalized functions 17, 10, 17]. A straightforward exercise shows that the functor $\mathcal{G}_{\mathcal{S}}(\cdot)$ defines a presheaf of differential algebras over $\mathbb{R}^{d}$ and a presheaf of modules over the factor ring of generalized constants $\overline{\mathbb{C}}=$ $\mathcal{E}_{M}(\mathbb{C}) / \mathcal{N}(\mathbb{C})$, with

$$
\begin{aligned}
\mathcal{E}_{M}(\mathbb{K}) & =\left\{\left(x_{\varepsilon}\right)_{\varepsilon} \in \mathbb{K}^{(0,1]}|\exists N \in \mathbb{N}, \quad| x_{\varepsilon} \mid=\mathrm{O}\left(\varepsilon^{-N}\right) \text { as } \varepsilon \rightarrow 0\right\} \\
\mathcal{N}(\mathbb{K}) & =\left\{\left(x_{\varepsilon}\right)_{\varepsilon} \in \mathbb{K}^{(0,1]}|\forall p \in \mathbb{N}, \quad| x_{\varepsilon} \mid=\mathrm{O}\left(\varepsilon^{p}\right) \text { as } \varepsilon \rightarrow 0\right\},
\end{aligned}
$$

for $\mathbb{K}=\mathbb{C}$ or $\mathbb{K}=\mathbb{R}, \mathbb{R}_{+}$. 
Set

$$
\mathcal{N}_{\mathcal{S}, *}(\Omega)=\left\{\left(f_{\varepsilon}\right)_{\varepsilon} \in \mathrm{C}^{\infty}(\Omega)^{(0,1]} \mid \forall q \in \mathbb{N}, \forall p \in \mathbb{N}, \mu_{q, 0}\left(f_{\varepsilon}\right)=\mathrm{O}\left(\varepsilon^{p}\right) \text { as } \varepsilon \rightarrow 0\right\} .
$$

We have the exact analogue of Theorems 1.2.25 and 1.2.27 of [1] (see [7]).

Proposition 1 If the open set $\Omega$ is a box, i.e. the product of $d$ open intervals of $\mathbb{R}$ (bounded or not) then $\mathcal{N}_{\mathcal{S}}(\Omega)=\mathcal{N}_{\mathcal{S}, *}(\Omega) \cap \mathcal{E}_{\mathcal{S}}(\Omega)$.

We shall need in the sequel some results concerning embeddings. Consider $\rho \in \mathcal{S}\left(\mathbb{R}^{d}\right)$ which satisfies

$$
\int \rho(x) \mathrm{d} x=1, \quad \int x^{\alpha} \rho(x) \mathrm{d} x=0 \text { for all } \alpha \in \mathbb{N}^{d} \backslash\{0\}
$$

and set

$$
\forall \varepsilon \in(0,1], \quad \forall x \in \mathbb{R}^{d}, \quad \rho_{\varepsilon}(x)=\varepsilon^{-d} \rho\left(x / \varepsilon^{-1}\right) .
$$

Proposition $2[7]$

(i) The map

$$
\sigma_{\mathcal{S}}: \quad \mathcal{S}\left(\mathbb{R}^{d}\right) \rightarrow \mathcal{G}_{\mathcal{S}}\left(\mathbb{R}^{d}\right), \quad f \mapsto\left[\left(f_{\varepsilon}\right)_{\varepsilon}\right]_{\mathcal{S}} \quad \text { with } f_{\varepsilon}=f \text { for all } \varepsilon \in(0,1]
$$

is an embedding of differential algebras.

(ii) The map

$$
\iota_{\mathcal{S}}: \quad \mathcal{O}_{C}^{\prime}\left(\mathbb{R}^{d}\right) \rightarrow \mathcal{G}_{\mathcal{S}}\left(\mathbb{R}^{d}\right), \quad u \mapsto\left(u * \rho_{\varepsilon}\right)_{\varepsilon}+\mathcal{N}_{\mathcal{S}}\left(\mathbb{R}^{d}\right)
$$

is an embedding of differential vector spaces. $\left(\mathcal{O}_{C}^{\prime}\left(\mathbb{R}^{d}\right)\right.$ denotes the space of rapidly decreasing distributions.)

(iii) Moreover, $\iota_{\mathcal{S} \mid \mathcal{S}\left(\mathbb{R}^{d}\right)}=\sigma_{\mathcal{S}}$.

\subsection{Temperate generalized functions}

Set

$$
\begin{gathered}
\mathcal{O}_{M}(\Omega)=\left\{f \in \mathrm{C}^{\infty}(\Omega) \mid \forall l \in \mathbb{N}, \exists q \in \mathbb{N}, \quad \mu_{-q, l}(f)<+\infty\right\} \\
\mathcal{E}_{\tau}(\Omega)=\left\{\left(f_{\varepsilon}\right)_{\varepsilon} \in \mathcal{O}_{M}(\Omega)^{(0,1]} \mid \forall l \in \mathbb{N}, \exists q \in \mathbb{N}, \exists N \in \mathbb{N}, \quad \mu_{-q, l}\left(f_{\varepsilon}\right)=\mathrm{O}\left(\varepsilon^{-N}\right) \text { as } \varepsilon \rightarrow 0\right\} \\
\mathcal{N}_{\tau}(\Omega)=\left\{\left(f_{\varepsilon}\right)_{\varepsilon} \in \mathcal{O}_{M}(\Omega)^{(0,1]} \mid \forall l \in \mathbb{N}, \exists q \in \mathbb{N}, \forall p \in \mathbb{N}, \quad \mu_{-q, l}\left(f_{\varepsilon}\right)=\mathrm{O}\left(\varepsilon^{p}\right) \text { as } \varepsilon \rightarrow 0\right\},
\end{gathered}
$$

where $\left(\mu_{r, l}\right)_{(r, l) \in \mathbb{Z} \times \mathbb{N}}$ are defined by (1).

The set $\mathcal{O}_{M}(\Omega)$ is the algebra of multiplicators (or of $\mathrm{C}^{\infty}$ functions with slow growth). One can show that $\mathcal{E}_{\tau}(\Omega)$ is a subalgebra of $\mathcal{O}_{M}(\Omega)^{(0,1]}$ and $\mathcal{N}_{\tau}(\Omega)$ an ideal of $\mathcal{E}_{\tau}(\Omega)$. The algebra $\mathcal{G}_{\tau}(\Omega)=\mathcal{E}_{\tau}(\Omega) / \mathcal{N}_{\tau}(\Omega)$ is called the algebra of tempered generalized functions [4, 11, 18. As for the case of $\mathcal{G}_{\mathcal{S}}(\cdot)$, the functor $\mathcal{G}_{\tau}(\cdot)$ defines a presheaf over $\mathbb{R}^{d}$ of differential algebras and a presheaf of modules over the factor ring $\overline{\mathbb{C}}$ introduced in subsection 2.1

The following result will be useful in the sequel. Set

$$
\mathcal{N}_{\tau, *}(\Omega)=\left\{\left(f_{\varepsilon}\right)_{\varepsilon} \in \mathcal{O}_{M}(\Omega)^{(0,1]} \mid \exists q \in \mathbb{N}, \forall p \in \mathbb{N}, \quad \mu_{-q, 0}\left(f_{\varepsilon}\right)=\mathrm{O}\left(\varepsilon^{p}\right) \text { as } \varepsilon \rightarrow 0\right\} .
$$

Proposition 3 [11] If the open set $\Omega$ is a box (see proposition [1]) then $\mathcal{N}_{\tau}(\Omega)=\mathcal{N}_{\tau, *} \cap \mathcal{E}_{\tau}(\Omega$ ).

The results about embeddings concerning $\mathcal{G}_{\tau}\left(\mathbb{R}^{d}\right)$ can be summarized (Theorems 1.2.27 and 1.2.28 of [1]) in the following proposition: 
Proposition 4 Consider $\rho \in \mathcal{S}\left(\mathbb{R}^{d}\right)$ which satisfies (3) and define $\left(\rho_{\varepsilon}\right)_{\varepsilon}$ as in (4).

(i) The map

$$
\sigma_{\tau}: \quad \mathcal{O}_{M}\left(\mathbb{R}^{d}\right) \rightarrow \mathcal{G}_{\tau}\left(\mathbb{R}^{d}\right), \quad f \mapsto(f)_{\varepsilon}+\mathcal{N}_{\tau}\left(\mathbb{R}^{d}\right), \quad \text { with } f_{\varepsilon}=f \text { for all } \varepsilon \in(0,1]
$$

is an embedding of differential algebras.

(ii) The map

$$
\iota_{\tau}: \quad \mathcal{S}^{\prime}\left(\mathbb{R}^{d}\right) \rightarrow \mathcal{G}_{\tau}\left(\mathbb{R}^{d}\right), \quad u \mapsto\left(u * \rho_{\varepsilon}\right)_{\varepsilon}+\mathcal{N}_{\tau}\left(\mathbb{R}^{d}\right)
$$

is an embedding of differential vector spaces.

(iii) Moreover, $\iota_{\tau \mid \mathcal{O}_{C}\left(\mathbb{R}^{d}\right)}=\sigma_{\tau}$, where

$$
\mathcal{O}_{C}(\Omega)=\left\{f \in \mathrm{C}^{\infty}(\Omega) \mid \exists q \in \mathbb{N}, \forall l \in \mathbb{N}, \quad \mu_{-q, l}(f)<+\infty\right\}
$$

\subsection{Regular subalgebras of $\mathcal{G}_{\mathcal{S}}(\Omega)$ and $\mathcal{G}_{\tau}(\Omega)$}

In the sequel, we need to consider some subspaces of $\mathcal{G}_{\mathcal{S}}(\cdot)$ and $\mathcal{G}_{\tau}(\cdot)$ with restrictive conditions of growth with respect to $\varepsilon^{-1}$. These spaces give a good framework for the extension of linear maps and for the convolution of generalized functions. These are essential properties for our main result. This notions are new for $\mathcal{G}_{\tau}(\Omega)$, but the main ideas have been given (for the purpose of microlocal analysis) for $\mathcal{G}(\cdot)$ and $\mathcal{G}_{\mathcal{S}}(\cdot)$ in [6, 7].

Definition 5 A non empty subspace $\mathcal{R}$ of $\mathbb{R}_{+}^{\mathbb{N}}$ is regular if

(i) $\mathcal{R}$ is "overstable" by translations and by maximum

$$
\begin{gathered}
\forall N \in \mathcal{R}, \forall k \in \mathbb{N}, \exists N^{\prime} \in \mathcal{R}, \forall n \in \mathbb{N}, \quad N(n)+k \leq N^{\prime}(n), \\
\forall N_{1} \in \mathcal{R}, \forall N_{2} \in \mathcal{R}, \exists N \in \mathcal{R}, \forall n \in \mathbb{N}, \quad \max \left(N_{1}(n), N_{2}(n)\right) \leq N(n) ;
\end{gathered}
$$

(ii) For all $N_{1}$ and $N_{2}$ in $\mathcal{R}$, there exists $N \in \mathcal{R}$ such that

$$
\forall\left(l_{1}, l_{2}\right) \in \mathbb{N}^{2}, \quad N_{1}\left(l_{1}\right)+N_{2}\left(l_{2}\right) \leq N\left(l_{1}+l_{2}\right) .
$$

For example, the set $\mathbb{R}_{+}^{\mathbb{N}}$ of all positive valued sequences and the set $\mathcal{B}$ of bounded sequences are regular.

Let $\mathcal{R}$ be a regular subset of $\mathbb{R}_{+}^{\mathbb{N}}$ and set

$$
\begin{gathered}
\mathcal{E}_{\mathcal{S}}^{\mathcal{R}}(\Omega)=\left\{\left(f_{\varepsilon}\right) \in \mathcal{E}(\Omega) \mid \exists N \in \mathcal{R}, \forall q \in \mathbb{N}, \forall l \in \mathbb{N}, \quad \mu_{q, l}\left(f_{\varepsilon}\right)=\mathrm{O}\left(\varepsilon^{-N(l)}\right)\right\} \\
\mathcal{E}_{\tau}^{\mathcal{R}}(\Omega)=\left\{\left(f_{\varepsilon}\right) \in \mathcal{E}(\Omega) \mid \exists N \in \mathcal{R}, \exists q \in \mathbb{N}, \forall l \in \mathbb{N}, \quad \mu_{-q, l}\left(f_{\varepsilon}\right)=\mathrm{O}\left(\varepsilon^{-N(l)}\right)\right\} .
\end{gathered}
$$

\section{Proposition 6}

(i) For all regular subspace $\mathcal{R}$ of $\mathbb{R}_{+}^{\mathbb{N}}, \mathcal{E}_{\mathcal{S}}^{\mathcal{R}}(\cdot)$ (resp. $\mathcal{E}_{\tau}^{\mathcal{R}}(\cdot)$ ) is a presheaf of differential algebras over the ring $\mathcal{E}_{M}(\mathbb{C})$.

(ii) For all regular subspaces $\mathcal{R}_{1}$ and $\mathcal{R}_{2}$ of $\mathbb{R}_{+}^{\mathbb{N}}$, with $\mathcal{R}_{1} \subset \mathcal{R}_{2}, \mathcal{E}_{\mathcal{S}}^{\mathcal{R}_{1}}(\cdot)$ (resp. $\mathcal{E}_{\tau}^{\mathcal{R}_{1}}(\cdot)$ ) is a subpresheaf of $\mathcal{E}_{\mathcal{S}}^{\mathcal{R}_{2}}(\cdot)$ (resp. $\left.\mathcal{E}_{\tau}^{\mathcal{R}_{2}}(\cdot)\right)$.

We refer the reader to $[\underline{6}$ for the proof for the case of rapidly decreasing generalized functions. The proof for temperate ones is similar.

Definition 7 For all regular subset $\mathcal{R}$ of $\mathbb{R}_{+}^{\mathbb{N}}$, the presheaf of factor algebras $\mathcal{G}_{\mathcal{S}}^{\mathcal{R}}(\cdot)$ (resp. $\mathcal{G}_{\tau}^{\mathcal{R}}(\cdot)$ ) is called the presheaf of $\mathcal{R}$-regular algebras of rapidly decreasing (resp. temperate) generalized functions.

Example 8 Taking $\mathcal{R}=\mathbb{R}_{+}^{\mathbb{N}}$, we recover the presheaf $\mathcal{G}_{\mathcal{S}}(\cdot)$ (resp. $\mathcal{G}_{\tau}(\cdot)$ ) of rapidly decreasing (resp. temperate) generalized functions. Taking $\mathcal{R}=\mathcal{B}$, we obtain the presheaf $\mathcal{G}_{\mathcal{S}}^{\infty}(\cdot)$ (resp. $\left.\mathcal{G}_{\tau}^{\infty}(\cdot)\right)$ which is analogue for $\mathcal{G}_{\mathcal{S}}(\cdot)$ (resp. $\left.\mathcal{G}_{\tau}(\cdot)\right)$ to the sheaf of $\mathcal{G}^{\infty}$-generalized functions for $\mathcal{G}(\cdot)$. (See [15] for $\mathcal{G}^{\infty}(\cdot)$ and [6] for $\left.\mathcal{G}_{\mathcal{S}}^{\infty}(\cdot).\right)$ 
Example 9 Rapidly decreasing and temperate generalized functions with slow asymptotic growth. We consider mainly the two following examples of regular spaces [5]:

$$
\mathcal{L}_{0}=\left\{q \in \mathbb{R}_{+}^{\mathbb{N}} \text { with } \lim _{l \rightarrow+\infty}(q(l) / l)=0\right\} \quad \mathcal{L}_{a}=\left\{q \in \mathbb{R}_{+}^{\mathbb{N}} \text { with } \lim _{l \rightarrow+\infty}(q(l) / l)<a\right\}(a>0) .
$$

The corresponding presheaves of algebras $\mathcal{G}_{\mathcal{S}}^{\mathcal{L}_{a}}(\cdot)$ (resp. $\left.\mathcal{G}_{\tau}^{\mathcal{L}_{a}}(\cdot)\right)$ are called the presheaves of rapidly decreasing (resp. temperate) generalized functions with slow asymptotic growth, with respect to the regularizing parameter $\varepsilon$.

\subsection{Fundamental lemma}

Lemma 10 Let a be a real in $[0,1]$. Consider $\rho \in \mathcal{S}\left(\mathbb{R}^{d}\right)$ which satisfies (3) and define $\left(\rho_{\varepsilon}\right)_{\varepsilon}$ as in (4). For any $\left(g_{\varepsilon}\right)_{\varepsilon} \in \mathcal{E}_{\mathcal{S}}^{\mathcal{L}_{a}}\left(\mathbb{R}^{d}\right)$ (resp. $\mathcal{E}_{\tau}^{\mathcal{L}_{a}}\left(\mathbb{R}^{d}\right)$ ), we have

$$
\left(g_{\varepsilon} * \rho_{\varepsilon}-g_{\varepsilon}\right)_{\varepsilon} \in \mathcal{N}_{\mathcal{S}}\left(\mathbb{R}^{d}\right) \quad\left(\text { resp. } \mathcal{N}_{\tau}\left(\mathbb{R}^{d}\right)\right) .
$$

Proof. It suffices to treat the case $a=1$, since $\mathcal{E}_{\mathcal{S}}^{\mathcal{L}_{a}} \subset \mathcal{E}_{\mathcal{S}}^{\mathcal{L}_{1}}$ (resp. $\mathcal{E}_{\tau}^{\mathcal{L}^{a}} \subset \mathcal{E}_{\tau}^{\mathcal{L}_{1}}$ ). We shall do the proof for the case $d=1$, the general case only differs by more complicated algebraic expressions.

(i) Case of $\mathcal{E}_{\mathcal{S}}^{\mathcal{L}_{1}}(\mathbb{R})_{\text {. }}$ Fix $\left(g_{\varepsilon}\right)_{\varepsilon} \in \mathcal{E}_{\mathcal{S}}^{\mathcal{L}_{1}}(\mathbb{R})$ and set, for $\varepsilon \in(0,1]$,

$$
\forall y \in \mathbb{R}, \quad \Delta_{\varepsilon}(y)=\left(g_{\varepsilon} * \rho_{\varepsilon}\right)(y)-g_{\varepsilon}(y)=\int\left(g_{\varepsilon}(y-x)-g_{\varepsilon}(y)\right) \rho_{\varepsilon}(x) \mathrm{d} x .
$$

There exists $N \in \mathcal{L}_{1}$ such that

$$
\forall q \in \mathbb{N}, \forall l \in \mathbb{N}, \forall \xi \in \mathbb{R}, \quad\left|g_{\varepsilon}^{(l)}(\xi)\right| \leq C_{l, q}(1+|\xi|)^{-q} \varepsilon^{-N(l)} \quad\left(C_{l, q}>0\right),
$$

for $\varepsilon$ smaller than some $\eta_{i, q}$ depending on $i$ and $q$.

Let $p$ and $q$ be two integers. As $\limsup _{l \rightarrow+\infty}(N(l) / l)<1$, we get $\lim _{l \rightarrow+\infty}(l-N(l))=+\infty$ and the existence of an integer $k$ such that $k-N(k)>p$. Taylor's formula gives

$$
\forall(x, y) \in \mathbb{R}^{2}, \quad g_{\varepsilon}(y-x)-g_{\varepsilon}(y)=\sum_{l=1}^{k-1} \frac{(-x)^{l}}{l !} g_{\varepsilon}^{(l)}(y)+\frac{(-x)^{k}}{(k-1) !} \int_{0}^{1} g_{\varepsilon}^{(k)}(y-u x)(1-u)^{k-1} \mathrm{~d} u,
$$

and

$$
\forall y \in \mathbb{R}, \quad \Delta_{\varepsilon}(y)=\int \frac{(-x)^{k}}{(k-1) !}\left(\int_{0}^{1} g_{\varepsilon}^{(k)}(y-u x)(1-u)^{k-1} \mathrm{~d} u\right) \rho_{\varepsilon}(x) \mathrm{d} x .
$$

since $\int x^{i} \theta_{\varepsilon}(x) \mathrm{d} x=0$ (for all $i \geq 1$ ). Setting $v=x / \varepsilon$, we get

$$
\forall y \in \mathbb{R}, \quad \Delta_{\varepsilon}(y)=\frac{\varepsilon^{k}}{(k-1) !} \int(-v)^{k}\left(\int_{0}^{1} g_{\varepsilon}^{(k)}(y-\varepsilon u v)(1-u)^{k-1} \mathrm{~d} u\right) \rho(v) \mathrm{d} v .
$$

and

$$
\forall y \in \mathbb{R}, \quad\left|\Delta_{\varepsilon}(y)\right| \leq \frac{\varepsilon^{k}}{(k-1) !} \int|v|^{k}\left(\int_{0}^{1}\left|g_{\varepsilon}^{(k)}(y-\varepsilon u v)\right| \mathrm{d} u\right)|\rho(v)| \mathrm{d} v .
$$

For all $y \in \mathbb{R}, \varepsilon \in\left(0, \eta_{k, q}\right], u \in(0,1]$, we have

$$
\left|g_{\varepsilon}^{(k)}(y-\varepsilon u v)\right| \leq C_{k, q}(1+|y-\varepsilon u v|)^{-q} \varepsilon^{-N(k)} .
$$

Since $\rho$ is rapidly decreasing, there exists $C>0$ such that

$$
\forall v \in \mathbb{R}, \quad|\rho(v)| \leq C(1+|v|)^{-q-k-2} .
$$


Replacing in (9), we get the existence of a constant $C_{k, q}^{\prime}>0$ such that

$$
\begin{aligned}
& \forall y \in \mathbb{R}, \forall \varepsilon \in\left(0, \eta_{k, q}\right], \quad\left|\Delta_{\varepsilon}(y)\right| \\
& \quad \leq C_{k, q}^{\prime} \varepsilon^{k-N(k)} \int\left(\int_{0}^{1}(1+|y-\varepsilon u v|)^{-q}(1+|v|)^{-q} \mathrm{~d} u\right)|v|^{k}(1+|v|)^{-k-2} \mathrm{~d} v .
\end{aligned}
$$

One can verify (by hand) that $(1+|y-\varepsilon u v|)^{-q}(1+|v|)^{-q} \leq(1+|y|)^{-q}$, for all $y \in \mathbb{R}, \varepsilon \in(0,1]$, $u \in(0,1]$. Thus, there exists a constant $C_{k, q}^{\prime \prime}>0$ such that

$$
\forall y \in \mathbb{R}, \forall \varepsilon \in\left(0, \eta_{k, q}\right], \quad\left|\Delta_{\varepsilon}(y)\right| \leq C_{k, q}^{\prime \prime} \varepsilon^{k-N(k)}(1+|y|)^{-q} .
$$

By assumption on $k$, we finally get

$$
\sup _{y \in \mathbb{R}}\left|(1+|y|)^{q} \Delta_{\varepsilon}(y)\right|=\mathrm{O}\left(\varepsilon^{p}\right) \text { as } \varepsilon \rightarrow 0 .
$$

Thus, $\Delta_{\varepsilon}(y)$ satisfies the 0 -estimate of the ideal $\mathcal{N}_{\mathcal{S}}(\mathbb{R})$. As $\left(\Delta_{\varepsilon}\right)_{\varepsilon} \in \mathcal{E}_{\mathcal{S}}(\mathbb{R})$, we can conclude that $\left(\Delta_{\varepsilon}\right)_{\varepsilon} \in \mathcal{N}_{\mathcal{S}}(\mathbb{R})$, without estimating the derivatives by using Proposition 1

(ii) Case of $\mathcal{E}_{\tau}^{\mathcal{L}_{1}}(\mathbb{R})$.- The proof is an improvement of the proof of Theorems 1.2.28 of [1], based on the ideas developed for the case of $\mathcal{E}_{S}^{\mathcal{L}_{1}^{1}}(\mathbb{R})$ above.

Remark 11 Consider a net of mollifiers $\left(\rho_{\varepsilon}\right)_{\varepsilon}$ as in Lemma 10 Relation (8) shows that $\left[\left(\rho_{\varepsilon}\right)_{\varepsilon}\right]_{\mathcal{S}}$ (resp. $\left.\left[\left(\rho_{\varepsilon}\right)_{\varepsilon}\right]_{\tau}\right)$ plays the role of identity for convolution in $\mathcal{G}_{\mathcal{S}}^{\mathcal{L}^{a}}\left(\mathbb{R}^{d}\right)$ (resp. $\mathcal{G}_{\tau}^{\mathcal{L}_{a}}\left(\mathbb{R}^{d}\right)$ ) whereas this is false for $\mathcal{G}_{\mathcal{S}}\left(\mathbb{R}^{d}\right)$ (resp. $\mathcal{G}_{\tau}\left(\mathbb{R}^{d}\right)$ ). This is an essential feature of these new spaces.

\section{$3 \quad$ Generalized integral operators}

As mentioned in the introduction, we consider here generalized integral operators acting on $\mathcal{G}_{\mathcal{S}}\left(\mathbb{R}^{n}\right)$ with values in $\mathcal{G}_{\tau}\left(\mathbb{R}^{m}\right)$. (We refer the reader to [2] and [10] for the more usual case of generalized integral operators acting on $\mathcal{G}\left(\mathbb{R}^{n}\right)$.) From now on $m$ and $n$ are two strictly positive integers.

Lemma 12 Consider $H \in \mathcal{G}_{\tau}\left(\mathbb{R}^{m+n}\right), f \in \mathcal{G}_{\mathcal{S}}\left(\mathbb{R}^{n}\right)$ and $\left(H_{\varepsilon}\right)_{\varepsilon}$ (resp. $\left.\left(f_{\varepsilon}\right)_{\varepsilon}\right)$ any representative of $H$ (resp. f). The net of $C^{\infty}$ maps

$$
\left(\widetilde{H}_{\varepsilon}\left(f_{\varepsilon}\right)\right)_{\varepsilon}:=\left(x \mapsto \int H_{\varepsilon}(x, y) f_{\varepsilon}(y) \mathrm{d} y\right)_{\varepsilon}
$$

belongs to $\mathcal{E}_{\tau}\left(\mathbb{R}^{n}\right)$ and the class $\left[\left(\widetilde{H}_{\varepsilon}\left(f_{\varepsilon}\right)\right)_{\varepsilon}\right]_{\tau}$ depends only on $H$ and $f$ but not on the representatives $\left(H_{\varepsilon}\right)_{\varepsilon}$ and $\left(f_{\varepsilon}\right)_{\varepsilon}$.

Proof. Firstly, for all $x \in \mathbb{R}^{m}$ and $\varepsilon \in(0,1], H_{\varepsilon}(x, \cdot) f_{\varepsilon}(\cdot)$ belongs to $L^{1}\left(\mathbb{R}^{n}\right)$ as well as its derivatives, since $f_{\varepsilon} \in \mathcal{S}\left(\mathbb{R}^{n}\right)$ and $H_{\varepsilon}(x, \cdot) \in \mathcal{O}_{M}\left(\mathbb{R}^{n}\right)$. Thus, $\widetilde{H}_{\varepsilon}\left(f_{\varepsilon}\right)$ is well defined and it is easily seen that $\widetilde{H}_{\varepsilon}\left(f_{\varepsilon}\right)$ belongs to $\mathrm{C}^{\infty}\left(\mathbb{R}^{m}\right)$. Secondly, for any $\alpha \in \mathbb{N}^{m}$, there exist $q_{1} \in \mathbb{N}$ and $C_{1}>0$ such that (for $\varepsilon$ small enough)

$$
\forall(x, y) \in \mathbb{R}^{m+n}, \quad\left|\partial_{x}^{\alpha} H_{\varepsilon}(x, y)\right| \leq C_{1}(1+|x|)^{q_{1}}(1+|y|)^{q_{1}} \varepsilon^{-q_{1}} .
$$

As $\left(f_{\varepsilon}\right)_{\varepsilon} \in \mathcal{E}_{\mathcal{S}}\left(\mathbb{R}^{n}\right)$, there exist $q_{2} \in \mathbb{N}$ and $C_{2}>0$ such that (for $\varepsilon$ small enough)

$$
\forall y \in \mathbb{R}^{n}, \quad\left|f_{\varepsilon}(y)\right| \leq C_{2}(1+|y|)^{-q_{1}-d-1} \varepsilon^{-q_{2}} .
$$

Inserting (11) and (12) in the definition of $\widetilde{H}_{\varepsilon}\left(f_{\varepsilon}\right)$, we get a constant $C_{3}>0$ such that

$$
\forall x \in \mathbb{R}^{m}, \quad\left|\partial^{\alpha} \widetilde{H}_{\varepsilon}\left(f_{\varepsilon}\right)(x)\right| \leq C_{3}(1+|x|)^{q_{1}} \varepsilon^{-q_{1}-q_{2}} .
$$


Thus $\left(\widetilde{H}_{\varepsilon}\right)_{\varepsilon}$ is in $\mathcal{E}_{\tau}\left(\mathbb{R}^{m}\right)$. Finally, suppose that $\left(H_{\varepsilon}\right)_{\varepsilon}$ (resp. $\left.\left(f_{\varepsilon}\right)_{\varepsilon}\right)$ is in $\mathcal{N}_{\tau}\left(\mathbb{R}^{m+n}\right)$ (resp. $\mathcal{N}_{\mathcal{S}}\left(\mathbb{R}^{n}\right)$ ). From estimates similar to (11) and (12), we get that $\left(\widetilde{H}_{\varepsilon}\left(f_{\varepsilon}\right)\right)_{\varepsilon}$ is in $\mathcal{N}_{\tau}\left(\mathbb{R}^{m}\right)$. Hence, the independence on the representatives of $\left[\left(\widetilde{H}_{\varepsilon}\left(f_{\varepsilon}\right)\right)_{\varepsilon}\right]_{\tau}$ is proved.

Lemma 12 justifies the following:

Definition 13 Let $H$ be in $\mathcal{G}_{\tau}\left(\mathbb{R}^{m+n}\right)$. The integral operator of kernel $H$ is the map $\widetilde{H}$ defined by

$$
\widetilde{H}: \quad \mathcal{G}_{\mathcal{S}}\left(\mathbb{R}^{n}\right) \rightarrow \mathcal{G}_{\tau}\left(\mathbb{R}^{m}\right), \quad f \mapsto \widetilde{H}(f)=\left[\left(x \mapsto \int H_{\varepsilon}(x, y) f_{\varepsilon}(y) \mathrm{d} y\right)_{\varepsilon}\right]_{\tau},
$$

with the notations of Lemma 12.

Proposition 14 With the notations of Definition 13, the operator $\widetilde{H}$ defines a linear mapping from $\mathcal{G}_{\tau}\left(\mathbb{R}^{n}\right)$ to $\mathcal{G}_{\mathcal{S}}\left(\mathbb{R}^{m}\right)$. Moreover, the map

$$
\mathcal{G}_{\tau}\left(\mathbb{R}^{m+n}\right) \rightarrow \mathbf{L}\left(\mathcal{G}_{\mathcal{S}}\left(\mathbb{R}^{n}\right), \mathcal{G}_{\tau}\left(\mathbb{R}^{m}\right)\right), \quad H \mapsto \widetilde{H}
$$

is injective.

Proof. Only the last assertion needs a proof. Consider $H \in \mathcal{G}_{\tau}\left(\mathbb{R}^{m+n}\right)$ and let $\left(H_{\varepsilon}\right)_{\varepsilon}$ be one of its representative. As $\left(H_{\varepsilon}\right)_{\varepsilon}$ is in $\mathcal{E}_{\tau}\left(\mathbb{R}^{m+n}\right)$, there exist $q \in \mathbb{N}$ and $C_{0}>0$ such that

$$
\forall(x, y) \in \mathbb{R}^{m+n}, \quad\left|H_{\varepsilon}(x, y)\right| \leq C_{0}\left(1+|(x, y)|^{2}\right)^{q} \varepsilon^{-q} \leq C_{0}\left(\left(1+|x|^{2}\right)\left(1+|y|^{2}\right)\right)^{q} \varepsilon^{-q},
$$

for $\varepsilon$ small enough. Now, suppose that $\widetilde{H}$ is null. Then, for all $g \in \mathcal{G}_{\mathcal{S}}\left(\mathbb{R}^{n}\right)$ with representative $\left(g_{\varepsilon}\right)_{\varepsilon}$, there exists $r>0$ such that

$$
\forall p \in \mathbb{N}, \exists C>0, \forall x \in \mathbb{R}^{m}, \quad\left|\widetilde{H}_{\varepsilon}\left(g_{\varepsilon}\right)(x)\right|=\left|\int H_{\varepsilon}(x, y) g_{\varepsilon}(y) \mathrm{d} y\right| \leq C\left(1+|x|^{2}\right)^{r} \varepsilon^{p},
$$

for $\varepsilon$ small enough.

Set, for all $(x, y) \in \mathbb{R}^{m+n}, B_{\varepsilon}(x, y)=\varepsilon^{q} H_{\varepsilon}(x, y)\left(\left(1+|x|^{2}\right)\left(1+|y|^{2}\right)\right)^{-q-1}$. The net $\left(B_{\varepsilon}\right)_{\varepsilon}$ belongs to $\mathcal{E}_{\tau}\left(\mathbb{R}^{m+n}\right)$. For $f \in \mathcal{G}_{\mathcal{S}}\left(\mathbb{R}^{n}\right)$ with representative $\left(f_{\varepsilon}\right)_{\varepsilon}$, we have

$$
\int B_{\varepsilon}(x, y) f_{\varepsilon}(y) \mathrm{d} y=\left(1+|x|^{2}\right)^{-q-1} \int H_{\varepsilon}(x, y) \varepsilon^{q}\left(1+|y|^{2}\right)^{-q-1} f_{\varepsilon}(y) \mathrm{d} y .
$$

As the net of functions $\left(y \mapsto \varepsilon^{q}\left(1+|y|^{2}\right)^{-q-1} f_{\varepsilon}(y)\right)_{\varepsilon}$ belongs to $\mathcal{E}_{\mathcal{S}}\left(\mathbb{R}^{n}\right)$, we get from (13), the existence of $r_{1} \in \mathbb{Z}\left(r_{1}=r-q-1\right)$ such that

$$
\forall p \in \mathbb{N}, \exists C>0, \forall x \in \mathbb{R}^{m}, \quad\left|\widetilde{B}_{\varepsilon}\left(f_{\varepsilon}\right)(x)\right|=\left|\int B_{\varepsilon}(x, y) f_{\varepsilon}(y) \mathrm{d} y\right| \leq C\left(1+|x|^{2}\right)^{r_{1}} \varepsilon^{p},
$$

for $\varepsilon$ small enough. Thus, the operator $\widetilde{B}$ defined by $B=\left[\left(B_{\varepsilon}\right)_{\varepsilon}\right]_{\tau}$ is null.

Remark that it suffices to show that

$$
\forall p \in \mathbb{N}, \exists \eta^{\prime}>0, \exists C^{\prime}>0, \forall \varepsilon \in\left(0, \eta^{\prime}\right], \forall(x, y) \in \mathbb{R}^{m+n}, \quad\left|B_{\varepsilon}(x, y)\right| \leq C^{\prime} \varepsilon^{p}
$$

to conclude that $H$ is null. Indeed, if (15) holds, for any $p \in \mathbb{N}$, we get a constant $C^{\prime}>0$ such that

$$
\forall(x, y) \in \mathbb{R}^{m+n},\left|H_{\varepsilon}(x, y)\right| \leq \varepsilon^{p-q} C^{\prime}\left(\left(1+|x|^{2}\right)\left(1+|y|^{2}\right)\right)^{q+1} \leq \varepsilon^{p-q} C^{\prime}\left(1+|(x, y)|^{2}\right)^{2 q+2},
$$

for $\varepsilon$ small enough. As $q$ is fixed, this proves that $\left(H_{\varepsilon}\right)_{\varepsilon}$ satisfies the 0-estimate of $\mathcal{N}_{\tau}\left(\mathbb{R}^{m+n}\right)$.

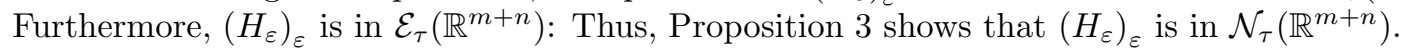


We are now going to show (15). We suppose, on the contrary, that

$$
\exists p_{0} \in \mathbb{N}, \forall \eta^{\prime}>0, \forall C^{\prime}>0, \exists \varepsilon \in\left(0, \eta^{\prime}\right], \exists(x, y) \in \mathbb{R}^{m+n}, \quad\left|B_{\varepsilon}(x, y)\right|>C^{\prime} \varepsilon^{p_{0}} .
$$

Thus, there exists a sequence $\left(\varepsilon_{l}\right)_{l}$ converging to 0 such that, for all $l \in \mathbb{N}$, there exists $\left(x_{l}, y_{l}\right) \in$ $\mathbb{R}^{m+n}$ such that $\left|B_{\varepsilon_{l}}\left(x_{l}, y_{l}\right)\right| \geq \varepsilon_{l}^{p_{0}}$.

As

$$
\forall \varepsilon \in(0,1], \quad \forall(x, y) \in \mathbb{R}^{m+n}, \quad\left|B_{\varepsilon}(x, y)\right| \leq C_{0}\left(1+|x|^{2}\right)^{-1}\left(1+|y|^{2}\right)^{-1},
$$

the function $B_{\varepsilon}$ is bounded by $C_{0}$, with $\lim _{|(x, y)| \rightarrow+\infty}\left|B_{\varepsilon}(x, y)\right|=0$. Thus, there exists $\left(x_{\varepsilon}, y_{\varepsilon}\right) \in$ $\mathbb{R}^{m+n}$, such that $\sup _{(x, y) \in \mathbb{R}^{m+n}}\left|B_{\varepsilon}(x, y)\right|=\left|B_{\varepsilon}\left(x_{\varepsilon}, y_{\varepsilon}\right)\right|=M_{\varepsilon}$. Moreover, due to (16), we have

$$
\forall l \in \mathbb{N}, \quad M_{\varepsilon_{l}}>\varepsilon_{l}^{p_{0}} .
$$

Note that we necessarily have

$$
\forall l \in \mathbb{N}, \quad\left|x_{\varepsilon_{l}}\right| \leq \varepsilon^{-p_{0}} \text { and }\left|y_{\varepsilon_{l}}\right| \leq \varepsilon^{-p_{0}} .
$$

(Indeed, supposing that: $\exists l \in \mathbb{N},\left|x_{\varepsilon_{l}}\right|>\varepsilon_{l}^{-p_{0}}$ or $\left|y_{\varepsilon}\right|>\varepsilon_{l}^{-p_{0}}$ contradicts (18), using (17).)

For all $l \in \mathbb{N}$, we can find a neighborhood $V_{l}$ (resp. $W_{l}$ ) of $x_{\varepsilon_{l}}$ (resp. $y_{\varepsilon_{l}}$ ) such that

$$
\forall(x, y) \in V_{l} \times W_{l}, \quad\left|B_{\varepsilon}(x, y)\right| \geq M_{\varepsilon_{l}} / 2 .
$$

Moreover, for all $l \in \mathbb{N}, V_{l}$ (resp. $W_{l}$ ) can be chosen such that its diameter $\delta\left(W_{l}\right)$ is greater than some $\varepsilon_{l}^{p_{1}}$, for a fixed $p_{1} \geq 0$. Indeed, as the net $\left(B_{\varepsilon}\right)_{\varepsilon}$ belongs to $\mathcal{E}_{\tau}\left(\mathbb{R}^{m+n}\right)$ and as $\left(x_{\varepsilon_{l}}, y_{\varepsilon_{l}}\right)_{\varepsilon}$ satisfies (19), the differential of $B_{\varepsilon_{l}}$ grows at most polynomially in $\varepsilon_{l}^{-1}$ for $l \rightarrow+\infty$ in some convex neighborhood of $\left(x_{\varepsilon_{l}}, y_{\varepsilon_{l}}\right)_{\varepsilon}$ of diameter, let us say, 1. Thus, supposing that: $\forall s \in \mathbb{N}, \exists l \in \mathbb{N}$, $\delta\left(W_{l}\right) \leq \varepsilon_{l}^{s}$ leads to a contradiction, since it violates the growth property of the sequence $\left(\nabla B_{\varepsilon_{l}}\right)_{l}$.

We define a net $\left(\theta_{\varepsilon}\right)_{\varepsilon}$ as follows. For all $l \in \mathbb{N}, \theta_{\varepsilon_{l}} \in \mathcal{D}\left(\mathbb{R}^{n}\right)$ is such that $0 \leq \theta_{\varepsilon_{l}} \leq 1, \theta_{\varepsilon} \equiv 1$ on $W_{l}$. For $\varepsilon \notin\left\{\varepsilon_{l}, l \in \mathbb{N}\right\}$ we simply choose $\theta_{\varepsilon} \equiv 0$. Moreover, we can suppose that the net $\left(\theta_{\varepsilon}\right)_{\varepsilon}$ is in $\mathcal{E}_{\mathcal{S}}\left(\mathbb{R}^{n}\right)$. (This is done by starting from some $\theta \in \mathcal{D}\left(\mathbb{R}^{n}\right)$ with $0 \leq \theta \leq 1, \theta \equiv 1$ on $B(0,1)$ and then by using linear transformations.) Set, for all $\varepsilon \in(0,1]$ and $y \in \mathbb{R}^{n}, f_{\varepsilon}(y)=\overline{B_{\varepsilon}\left(x_{\varepsilon}, y\right)} \theta_{\varepsilon}(y)$. As the net $\left(B_{\varepsilon}\right)_{\varepsilon}$ belongs to $\mathcal{E}_{\tau}\left(\mathbb{R}^{n}\right)$ and $\left(x_{\varepsilon_{l}}\right)_{l}$ satisfies (19), the net $\left(f_{\varepsilon}\right)_{\varepsilon}$ is in $\mathcal{E}_{\mathcal{S}}\left(\mathbb{R}^{n}\right)$. Therefore, using (14), we get the existence of $r_{1}$ - which can always be supposed positive - such that

$$
\forall p \in \mathbb{N}, \exists \eta>0, \exists C>0, \forall x \in \mathbb{R}^{m}, \forall \varepsilon \in(0, \eta], \quad\left|\widetilde{B}_{\varepsilon}\left(f_{\varepsilon}\right)(x)\right| \leq C\left(1+|x|^{2}\right)^{r_{1}} \varepsilon^{p},
$$

Returning to the definition of $\left(W_{l}\right)_{l}$ and $\left(\theta_{\varepsilon_{l}}\right)_{l}$, we have, for all $l \in \mathbb{N}$,

$$
\begin{aligned}
\left|\widetilde{B}_{\varepsilon_{l}}\left(f_{\varepsilon_{l}}\right)\left(x_{\varepsilon_{l}}\right)\right|=\int\left|B_{\varepsilon_{l}}\left(x_{\varepsilon_{l}}, y\right)\right|^{2} \theta_{\varepsilon_{l}}(y) \mathrm{d} y & \geq \int_{W_{l}}\left|B_{\varepsilon_{l}}\left(x_{\varepsilon_{l}}, y\right)\right|^{2} \mathrm{~d} y \\
& \geq \delta\left(W_{l}\right) M_{\varepsilon_{l}}^{2} / 4 \geq \varepsilon_{l}^{p_{1}} M_{\varepsilon_{l}}^{2} / 4 .
\end{aligned}
$$

Inserting (21) in (20), we get

$$
\forall p \in \mathbb{N}, \quad \exists C>0, \quad M_{\varepsilon_{l}}^{2} \leq 4 C\left(1+\left|x_{\varepsilon_{l}}\right|^{2}\right)^{r_{1}} \varepsilon_{l}^{p-p_{1}},
$$

for $l$ large enough. From (19), we have $\left(1+\left|x_{\varepsilon_{l}}\right|^{2}\right)^{r_{1}} \leq\left(1+\varepsilon_{l}^{-2 p_{0}}\right)^{r_{1}} \leq 2^{r_{1}} \varepsilon_{l}^{-2 p_{0} r_{1}}$, for all $l \in \mathbb{N}$. Finally, by setting $p_{2}=2 p_{0} r_{1}+p_{1}$, we get

$$
\forall p \in \mathbb{N}, \quad \exists C^{\prime}>0, \quad M_{\varepsilon_{l}} \leq C^{\prime} \varepsilon_{l}^{\left(p-p_{2}\right) / 2},
$$

for $l$ large enough. Thus, we get a contradiction with (18), ending the proof. 


\section{Kernel Theorems}

\subsection{Extension of linear maps}

Nets of maps $\left(L_{\varepsilon}\right)_{\varepsilon}$ between two topological algebras, having some good growth properties with respect to the parameter $\varepsilon$, can be extended to act between the corresponding Colombeau algebras, as it is shown in 51,11, 18, for example. We are going to introduce here new notions adapted to our framework. In the sequel $\mathcal{L}(\cdot, \cdot)($ resp. $\mathbf{L}(\cdot, \cdot))$ denote a space of continuous linear maps acting between classical spaces (resp. of $\overline{\mathbb{C}}$ linear maps acting between generalized spaces).

Definition 15 Let $j$ be an integer and $\left(L_{\varepsilon}\right)_{\varepsilon} \in \mathcal{L}\left(\mathcal{S}\left(\mathbb{R}^{n}\right), \mathcal{O}_{M}\left(\mathbb{R}^{m}\right)\right)^{(0,1]}$ be a net of linear maps.

(i) We say that $\left(L_{\varepsilon}\right)_{\varepsilon}$ is moderate (resp. negligible) if

$$
\begin{aligned}
& \forall l \in \mathbb{N}, \exists\left(C_{\varepsilon}\right)_{\varepsilon} \in \mathcal{E}_{M}\left(\mathbb{R}_{+}\right)\left(\text {resp. } \mathcal{N}\left(\mathbb{R}_{+}\right)\right), \exists\left(p, q, l^{\prime}\right) \in \mathbb{N}^{3}, \\
& \forall f \in \mathcal{S}\left(\mathbb{R}^{n}\right), \quad \mu_{-p, l}\left(L_{\varepsilon}(f)\right) \leq C_{\varepsilon} \mu_{q, l^{\prime}}(f), \text { for } \varepsilon \text { small enough } .
\end{aligned}
$$

(ii) Let $(b, c)$ be in $[0,+\infty] \times \mathbb{R}_{+}$. We say that $\left(L_{\varepsilon}\right)_{\varepsilon}$ is $\mathcal{L}_{b, c}$-strongly moderate if

$$
\begin{aligned}
& \exists \lambda \in \mathcal{L}_{b}, \exists r \in \mathcal{L}_{c}, \forall l \in \mathbb{N}, \exists C \in \mathbb{R}_{+}, \quad \exists(p, q) \in \mathbb{N}^{2}, \\
& \forall f \in \mathcal{S}\left(\mathbb{R}^{n}\right), \quad \mu_{-p, l}\left(L_{\varepsilon}(f)\right) \leq C \varepsilon^{-r(l)} \mu_{q, \lambda(l)}(f), \text { for } \varepsilon \text { small enough. }
\end{aligned}
$$
(22).

For the strong moderation, more precise estimates are given for the constants which appear in

\section{Proposition 16}

(i) Any moderate net $\left(L_{\varepsilon}\right)_{\varepsilon} \in\left(\mathcal{L}\left(\mathcal{S}\left(\mathbb{R}^{n}\right), \mathcal{O}_{M}\left(\mathbb{R}^{m}\right)\right)\right)^{(0,1]}$ can be extended to a map L belonging to $\mathbf{L}\left(\mathcal{G}_{\mathcal{S}}\left(\mathbb{R}^{n}\right), \mathcal{G}_{\tau}\left(\mathbb{R}^{m}\right)\right)$ and defined by

$$
L(f)=\left(L_{\varepsilon}\left(f_{\varepsilon}\right)\right)_{\varepsilon}+\mathcal{N}_{\tau}\left(\mathbb{R}^{m}\right),
$$

where $\left(f_{\varepsilon}\right)_{\varepsilon}$ is any representative of $f$.

(ii) The extension $L$ depends only on the family $\left(L_{\varepsilon}\right)_{\varepsilon}$ in the following sense: If $\left(N_{\varepsilon}\right)_{\varepsilon}$ is a negligible net of maps, then the extensions of $\left(L_{\varepsilon}\right)_{\varepsilon}$ and $\left(L_{\varepsilon}+N_{\varepsilon}\right)_{\varepsilon}$ are equal.

(iii) If the family $\left(L_{\varepsilon}\right)_{\varepsilon}$ is moderate, with the assumption that the net of constants $\left(C_{\varepsilon}\right)_{\varepsilon}$ in (22) satisfies $C_{\varepsilon}=\mathrm{O}\left(\varepsilon^{-r(l)}\right)$ with $\lim \sup (r(l) / l)<c$, then $L\left(\mathcal{G}_{\mathcal{S}}^{\infty}\left(\mathbb{R}^{n}\right)\right)$ is included in $\mathcal{G}_{\tau}^{\mathcal{L}_{c}}\left(\mathbb{R}^{m}\right)$.

(iv) Let $(a, b, c)$ be in $\left(\mathbb{R}_{+}\right)^{3}$ : If the net $\left(L_{\varepsilon}\right)_{\varepsilon}$ is $\mathcal{L}_{b, c}$-strongly moderate, then $L\left(\mathcal{G}_{\mathcal{S}}^{\mathcal{L}_{a}}\left(\mathbb{R}^{n}\right)\right)$ is included in $\mathcal{G}_{\tau}^{\mathcal{L}_{a b+c}}\left(\mathbb{R}^{m}\right)$.

Moreover, $L\left(\mathcal{G}_{\mathcal{S}}^{\mathcal{L}_{0}}\left(\mathbb{R}^{n}\right)\right)$ is included in $\mathcal{G}_{\tau}^{\mathcal{L}_{c}}\left(\mathbb{R}^{m}\right)$ even if $b=+\infty$.

Proof. Assertions (i) \& (ii).- Fix $l \in \mathbb{N}$ and let $\left(f_{\varepsilon}\right)_{\varepsilon}$ be in $\mathcal{E}_{\mathcal{S}}\left(\mathbb{R}^{n}\right)$. According to the definition of moderate nets, we get $\left(C_{\varepsilon}\right)_{\varepsilon} \in \mathcal{E}_{M}\left(\mathbb{R}_{+}\right)$and $\left(p, q, l^{\prime}\right) \in \mathbb{N}^{3}$ such that

$$
\mu_{-p, l}\left(L_{\varepsilon}\left(f_{\varepsilon}\right)\right) \leq C_{\varepsilon} \mu_{q, l^{\prime}}\left(f_{\varepsilon}\right) \text {, for } \varepsilon \text { small enough. }
$$

Inequality (25) leads to $\left(L_{\varepsilon}\left(f_{\varepsilon}\right)\right)_{\varepsilon} \in \mathcal{E}_{\tau}\left(\mathbb{R}^{m}\right)$. Moreover, if $\left(f_{\varepsilon}\right)_{\varepsilon}$ belongs to $\mathcal{N}_{\mathcal{S}}\left(\mathbb{R}^{n}\right)$, the same inequality implies that $\left(L_{\varepsilon}\left(f_{\varepsilon}\right)\right)_{\varepsilon} \in \mathcal{N}_{\tau}\left(\mathbb{R}^{m}\right)$. These two properties show that $L$ is well defined by formula (24). The $\overline{\mathbb{C}}$ linearity follows from from the moderation. Moreover, inequality (25) implies easily the second assertion.

Assertion (iii) \& (iv).- We shall prove (iv) for $a \in(0,+\infty)$, since the proof of (iii) and of the case $a=0$ in (iv) are even simpler. Suppose that $\left(L_{\varepsilon}\right)_{\varepsilon}$ is $\mathcal{L}_{b, c}$-strongly moderate and consider $\left(f_{\varepsilon}\right)_{\varepsilon} \in \mathcal{E}_{\mathcal{S}}^{\mathcal{L}_{a}}\left(\mathbb{R}^{n}\right)$. There exist a sequence $\lambda \in \mathbb{R}_{+}^{\mathbb{N}}$, with $\limsup _{l \rightarrow+\infty}(\lambda(l) / l)<b$, and a sequence $r \in \mathbb{R}_{+}^{\mathbb{N}}$, with $\limsup _{l \rightarrow+\infty}(r(l) / l)<c$, such that

$$
\forall l \in \mathbb{N}, \exists C \in \mathbb{R}_{+}, \exists(p, q) \in \mathbb{N}^{2}, \quad \mu_{-p, l}\left(L_{\varepsilon}\left(f_{\varepsilon}\right)\right) \leq C \varepsilon^{-r(l)} \mu_{q, \lambda(l)}\left(f_{\varepsilon}\right) \text { (for } \varepsilon \text { small enough). }
$$


As $\left(f_{\varepsilon}\right)_{\varepsilon}$ is in $\mathcal{E}_{\mathcal{S}}^{\mathcal{L}_{a}}\left(\mathbb{R}^{n}\right)$, there exists a sequence $N \in \mathbb{R}_{+}^{\mathbb{N}}$, with $\limsup _{\lambda \rightarrow+\infty}(N(\lambda) / \lambda)<a$, such that

We get that

$$
\forall s \in \mathbb{N}, \forall \lambda \in \mathbb{N}, \quad \mu_{s, \lambda}\left(f_{\varepsilon}\right)=\mathrm{O}\left(\varepsilon^{-N(\lambda)}\right) \text { as } \varepsilon \rightarrow 0
$$

$$
\forall l \in \mathbb{N}, \exists C \in \mathbb{R}_{+}, \exists p \in \mathbb{N}, \quad \mu_{-p, l}\left(L_{\varepsilon}\left(f_{\varepsilon}\right)\right) \leq C \varepsilon^{-N_{1}(l)} \quad \text { with } N_{1}(l)=r(l)+N(\lambda(l)),
$$

for $\varepsilon$ small enough.

- If $\lambda(l)$ is bounded, we immediately have: $N_{1}(l) / l=\mathrm{O}(r(l) / l)$ for $l \rightarrow+\infty$.

- If $\lambda(l)$ is not bounded, for $\lambda(l) \neq 0$, we have

$$
\frac{N_{1}(l)}{l}=\frac{r(l)}{l}+\frac{N(\lambda(l))}{\lambda(l)} \frac{\lambda(l)}{l} .
$$

We have $\lim \sup _{l \rightarrow+\infty}(N(\lambda(l)) / \lambda(l))<a$ and thus $\lim \sup _{l \rightarrow+\infty} \frac{N(\lambda(l))}{\lambda(l)} \frac{\lambda(l)}{l}<a b$. This gives

$$
\limsup _{l \rightarrow+\infty}\left(N_{1}(l) / l\right)<a b+c
$$

and $\left(L_{\varepsilon}\left(f_{\varepsilon}\right)\right)_{\varepsilon} \in \mathcal{E}_{\tau}^{\mathcal{L}_{a b+c}}\left(\mathrm{C}^{\infty}\left(\mathbb{R}^{m}\right)\right)$, which shows the assertion.

Finally, if $\left(f_{\varepsilon}\right)_{\varepsilon}$ is in $\mathcal{E}_{\mathcal{S}}^{\mathcal{L}_{0}}\left(\mathbb{R}^{n}\right)$, the sequence $N$ can be chosen such that $\lim _{\lambda \rightarrow+\infty}(N(\lambda) / \lambda)=0$. Then, for $b=+\infty$, the sequence $l \mapsto \lambda(l) / l$ is bounded. It follows that $\limsup _{l \rightarrow+\infty}\left(N_{1}(l) / l\right)<c$.

\subsection{Main results}

Theorem 17 Consider $(a, b, c) \in \mathbb{R}_{+}^{3}$ with $a \leq 1$ and $a b+c \leq 1$. Let $\left(L_{\varepsilon}\right)_{\varepsilon} \in \mathcal{L}\left(\mathcal{S}\left(\mathbb{R}^{n}\right), \mathcal{O}_{M}\left(\mathbb{R}^{m}\right)\right)^{(0,1]}$ be a net of $\mathcal{L}_{b, c}$-strongly moderate linear maps and $L \in \mathbf{L}\left(\mathcal{G}_{\mathcal{S}}\left(\mathbb{R}^{n}\right), \mathcal{G}_{\tau}\left(\mathbb{R}^{m}\right)\right)$ its canonical extension. There exists $H_{L} \in \mathcal{G}_{\tau}\left(\mathbb{R}^{m+n}\right)$ such that

$$
\forall f \in \mathcal{G}_{\mathcal{S}}^{\mathcal{L}^{a}}\left(\mathbb{R}^{n}\right), \quad L(f)=\left[\left(x \longmapsto \int H_{L, \varepsilon}(x, y) f_{\varepsilon}(y) \mathrm{d} y\right)_{\varepsilon}\right],
$$

where $\left(H_{L, \varepsilon}\right)_{\varepsilon}$ (resp. $\left.\left(f_{\varepsilon}\right)_{\varepsilon}\right)$ is any representative of $H_{L}$ (resp. $\left.f\right)$.

Remark 18 In Theorem 17 the parameter b (resp. c) is related to the "regularity" of the net $\left(L_{\varepsilon}\right)_{\varepsilon}$, with respect to the derivative index $l$ in the family of semi-norms $\left(\mu_{r, l}\right)_{r, l}$ (resp. to the parameter $\varepsilon$ ). The more "irregular" the net of maps $\left(L_{\varepsilon}\right)_{\varepsilon}$ is, that is the bigger $b$ is (resp. the closer to $1 \mathrm{c}$ is), the smaller is the space on which equality (27) holds. The limit cases for c are $c=1$ (for which $a=0$ and 27) holds only on $\mathcal{G}_{\mathcal{S}}^{\mathcal{L}_{0}}\left(\mathbb{R}^{n}\right)$ ) and $c=0$ (the net of constants $\left(C_{\varepsilon}\right)_{\varepsilon}$ in relation (22) depends slowly on $\varepsilon)$ for which the conditions on $(a, b, c)$ are reduced to $a<1$ and $a b \leq 1$. (Note that these limiting conditions are induced by Lemma 10)

By using Proposition 16. (iii), we can obtain an analogon of Theorem 17valid for more irregular nets of maps.

Theorem 19 Let $\left(L_{\varepsilon}\right)_{\varepsilon} \in \mathcal{L}\left(\mathcal{S}\left(\mathbb{R}^{n}\right), \mathcal{O}_{M}\left(\mathbb{R}^{m}\right)\right)^{(0,1]}$ be a net of moderate linear maps such that the net of constants $\left(C_{\varepsilon}\right)_{\varepsilon}$ in relation [22) satisfies $C_{\varepsilon}=\mathrm{O}\left(\varepsilon^{-r(l)}\right)$ with $r \in \mathcal{L}_{1}$. Then, the extension $\left(L_{\varepsilon}\right)_{\varepsilon}$ admits an integral representation such that relation (27) holds for $f$ in $\mathcal{G}_{\mathcal{S}}^{\infty}\left(\mathbb{R}^{n}\right)$.

We turn now to the relationship with the classical isomorphism result: Consider

$$
\Lambda \in \mathcal{L}\left(\mathcal{S}\left(\mathbb{R}^{n}\right), \mathcal{S}^{\prime}\left(\mathbb{R}^{m}\right)\right)
$$

and define a net of linear mappings $\left(L_{\varepsilon}\right)_{\varepsilon}$ by

$$
L_{\varepsilon}: \quad \mathcal{S}\left(\mathbb{R}^{n}\right) \rightarrow \mathrm{C}^{\infty}\left(\mathbb{R}^{m}\right), \quad f \mapsto \Lambda(f) * \varphi_{\varepsilon^{s}}, \quad(s \text { fixed real parameter in }(0,1))
$$

where $\left(\varphi_{\varepsilon}\right)_{\varepsilon}$ is defined as in (4), starting from $\varphi \in \mathcal{S}\left(\mathbb{R}^{m}\right)$ which satisfies (3). We have: 


\section{Proposition 20}

(i) For all $\varepsilon \in(0,1], L_{\varepsilon}$ is continuous for the usual topologies of $\mathcal{S}\left(\mathbb{R}^{n}\right)$ and $\mathcal{O}_{M}\left(\mathbb{R}^{m}\right)$ and the net $\left(L_{\varepsilon}\right)_{\varepsilon}$ is $(0, s)$-strongly moderate.

(ii) From (i), the extension $L$ of the net $\left(L_{\varepsilon}\right)_{\varepsilon}$ admits a kernel $H_{L}$. Furthermore, for all $f \in \mathcal{S}\left(\mathbb{R}^{n}\right)$, $\Lambda(f)$ is equal to $\widetilde{H}_{L}(f)$ in the generalized distribution sense [14], that is

$$
\forall g \in \mathcal{S}\left(\mathbb{R}^{m}\right), \quad\langle\Lambda(f), g\rangle=\left\langle\widetilde{H}_{L}(f), g\right\rangle \text { in } \overline{\mathbb{C}} .
$$

In other words, equality (28) means that, for all $p \in \mathbb{N}$,

$$
\forall g \in \mathcal{S}\left(\mathbb{R}^{m}\right),\langle\Lambda(f), g\rangle-\int\left(\int H_{L, \varepsilon}(x, y) f(y) \mathrm{d} y\right) g(x) \mathrm{d} x=\mathrm{O}\left(\varepsilon^{p}\right), \text { as } \varepsilon \rightarrow 0,
$$

where $\left(H_{L, \varepsilon}\right)_{\varepsilon}$ is any representative of $H_{L}$. In particular, this result implies that $\Lambda(f)$ and $\widetilde{H}_{L}(f)$ are associated or weakly equal, i.e.

$$
\left(x \mapsto \int H_{L, \varepsilon}(x, y) f(y) \mathrm{d} y\right) \longrightarrow \Lambda(f) \text { in } \mathcal{S}^{\prime}\left(\mathbb{R}^{m}\right) \text { as } \varepsilon \rightarrow 0 .
$$

\section{Proofs of Theorem 17 and Proposition 20}

\subsection{Proof of Theorem 17}

We shall prove Theorem[17 (The proof of Theorem[19]follows the same lines.) Consider $\varphi \in \mathcal{S}\left(\mathbb{R}^{m}\right)$ (resp. $\psi \in \mathcal{S}\left(\mathbb{R}^{n}\right)$ ) which satisfies (3) and define $\left(\varphi_{\varepsilon}\right)_{\varepsilon}\left(\right.$ resp. $\left.\left(\psi_{\varepsilon}\right)_{\varepsilon}\right)$ as in (4). For all $\varepsilon \in(0,1]$ and $y \in \mathbb{R}^{n}$, we set

$$
\psi_{\varepsilon, .}: \quad \mathbb{R}^{n} \rightarrow \mathcal{S}\left(\mathbb{R}^{n}\right), \quad y \mapsto \psi_{\varepsilon, y}=\left\{v \mapsto \psi_{\varepsilon}(y-v)\right\} .
$$

Then, for all $\varepsilon \in(0,1]$, the map

$$
H_{\varepsilon}: \quad \mathbb{R}^{m+n} \rightarrow \mathbb{C}, \quad(x, y) \mapsto\left(L_{\varepsilon}\left(\psi_{\varepsilon, y}\right) * \varphi_{\varepsilon}\right)(x)=\int L_{\varepsilon}\left(\psi_{\varepsilon, y}\right)(x-u) \varphi_{\varepsilon}(u) \mathrm{d} u,
$$

is well defined.

Indeed, $L_{\varepsilon}\left(\psi_{\varepsilon, y}\right)$ belongs to $\mathcal{O}_{M}(\mathbb{R})$ and $\varphi_{\varepsilon}$ to $\mathcal{S}\left(\mathbb{R}^{m}\right)$, making $L_{\varepsilon}\left(\psi_{\varepsilon, y}\right)(x-\cdot) \varphi_{\varepsilon}(\cdot)$ - and its derivatives $-\mathrm{L}^{1}$ functions.

Lemma 21 For all $\varepsilon \in(0,1], H_{\varepsilon}$ is of class $\mathrm{C}^{\infty}$ and $\left(H_{\varepsilon}\right)_{\varepsilon} \in \mathcal{E}_{\tau}\left(\mathbb{R}^{m+n}\right)$.

Proof. The fact that $H_{\varepsilon}$ is of class $\mathrm{C}^{\infty}$ follows from classical arguments of integral calculus. It also uses the topological isomorphism between $\mathrm{C}^{\infty}\left(\mathbb{R}^{d_{1}}, \mathrm{C}^{\infty}\left(\mathbb{R}^{d_{2}}\right)\right)$ and $\mathrm{C}^{\infty}\left(\mathbb{R}^{d_{1}+d_{2}}, \mathbb{C}\right)\left(d_{1}\right.$, $d_{2} \in \mathbb{N} \backslash\{0\}$ ), the linearity and continuity of both $L_{\varepsilon}$ and the convolution. (See Lemma 28 and 29 in [5] for very close proofs.)

Let us now consider $(\alpha, \beta) \in \mathbb{N}^{m+n}$ and $\partial_{x}^{\alpha}$ (resp. $\partial_{y}^{\beta}$ ) the $\alpha$-partial derivative (resp. $\beta$-partial derivative) with respect to the variable $x$ (resp. y). and set $l=|\beta|$. We have

$$
\begin{aligned}
\forall(x, y) \in \mathbb{R}^{m+n}, \quad \partial_{x}^{\alpha} \partial_{y}^{\beta} H_{\varepsilon}(x, y) & =\left(\partial_{y}^{\beta} L_{\varepsilon}\left(\psi_{\varepsilon, y}\right) * \partial_{x}^{\alpha} \varphi_{\varepsilon}\right)(x) \\
& =\int \partial_{y}^{\beta} L_{\varepsilon}\left(\psi_{\varepsilon, y}\right)(x-u) \partial_{x}^{\alpha} \varphi_{\varepsilon}(u) \mathrm{d} u \\
& =\int \partial_{y}^{\beta} L_{\varepsilon}\left(\psi_{\varepsilon, y}\right)(x-\varepsilon \zeta) \partial_{x}^{\alpha} \varphi(\zeta) \mathrm{d} \zeta
\end{aligned}
$$

Using the moderation of $\left(L_{\varepsilon}\right)_{\varepsilon}$, we get the existence of $\left(C_{\varepsilon}\right)_{\varepsilon} \in \mathcal{E}_{M}\left(\mathbb{R}_{+}\right), \quad\left(p, q, l^{\prime}\right) \in \mathbb{N}^{3}$ such that, for $\varepsilon$ small enough,

$$
\begin{aligned}
\forall(x, \zeta) \in \mathbb{R}^{2 m}, \quad\left|\partial_{y}^{\beta} L_{\varepsilon}\left(\psi_{\varepsilon, y}\right)(x-u)\right| & \leq C_{\varepsilon}(1+|x-\varepsilon \zeta|)^{p} \mu_{q, l^{\prime}}\left(\psi_{\varepsilon, y}\right) \\
& \leq C_{\varepsilon}(1+|x|)^{p}(1+|\zeta|)^{p} \mu_{q, l^{\prime}}\left(\psi_{\varepsilon, y}\right) .
\end{aligned}
$$


We have

$$
\begin{aligned}
\mu_{q, l^{\prime}}\left(\psi_{\varepsilon, y}\right)=\sup _{w \in \mathbb{R}^{n},|\alpha| \leq l^{\prime}}(1+|y-w|)^{q}\left|\partial^{\alpha} \psi_{\varepsilon}(w)\right| \leq(1+|y|)^{q} \mu_{q, l^{\prime}} & \left(\psi_{\varepsilon}\right) \\
& \leq \varepsilon^{-n-l^{\prime}}(1+|y|)^{q} \mu_{q, l^{\prime}}(\psi) .
\end{aligned}
$$

Therefore, there exists $\left(C_{\varepsilon}^{\prime}\right)_{\varepsilon} \in \mathcal{E}_{M}\left(\mathbb{R}_{+}\right)$such that

$$
\forall(x, \zeta, y) \in \mathbb{R}^{2 m+n}, \quad\left|\partial_{y}^{\beta} L_{\varepsilon}\left(\psi_{\varepsilon, y}\right)(x-\varepsilon \zeta)\right| \leq C_{\varepsilon}^{\prime}(1+|x|)^{p}(1+|\zeta|)^{p}(1+|y|)^{q} .
$$

for $\varepsilon$ small enough. As $\partial_{x}^{\alpha} \varphi$ is rapidly decreasing, by replacing the last estimate above in (30), we get the existence of $C_{\varepsilon}^{\prime \prime} \in \mathcal{E}_{M}\left(\mathbb{R}_{+}\right)$such that

$$
\forall(x, y) \in \mathbb{R}^{m+n}, \quad\left|\partial_{x}^{\alpha} \partial_{y}^{\beta} H_{\varepsilon}(x, y)\right| \leq C_{\varepsilon}^{\prime \prime}(1+|x|)^{p}(1+|y|)^{q} \leq C_{\varepsilon}^{\prime \prime}(1+|(x, y)|)^{p+q},
$$

for $\varepsilon$ small enough. Thus, $\left(H_{\varepsilon}\right)_{\varepsilon} \in \mathcal{E}_{\tau}\left(\mathbb{R}^{m+n}\right)$ as claimed.

Lemma 22 For all $\left(f_{\varepsilon}\right)_{\varepsilon}$ in $\mathcal{E}_{S}\left(\mathbb{R}^{n}\right)$, we have

$$
\widetilde{H}_{\varepsilon}\left(f_{\varepsilon}\right)(x)=\left(L_{\varepsilon}\left(\psi_{\varepsilon} * f_{\varepsilon}\right) * \varphi_{\varepsilon}\right)(x) .
$$

Proof. Let $\left(f_{\varepsilon}\right)_{\varepsilon}$ be in $\mathcal{E}_{S}\left(\mathbb{R}^{n}\right)$. For any fixed $\varepsilon \in(0,1]$ and $x \in \mathbb{R}^{m}$, we have

$$
\widetilde{H}_{\varepsilon}\left(f_{\varepsilon}\right)(x)=\int\left(\int L_{\varepsilon}\left(\psi_{\varepsilon, y}\right)(x-u) \varphi_{\varepsilon}(u) \mathrm{d} u\right) f_{\varepsilon}(y) \mathrm{d} y .
$$

Using a similar argument as in the proof of Lemma 21] we get the existence of $C_{\varepsilon}(x)>0$ such that

$$
\forall u \in \mathbb{R}^{m}, \quad\left|L_{\varepsilon}\left(\psi_{\varepsilon, y}\right)(x-u)\right| \leq C_{\varepsilon}(x)(1+|u|)^{p} .
$$

Thus, the map $(u, y) \mapsto L_{\varepsilon}\left(\psi_{\varepsilon, y}\right)(x-u) \varphi_{\varepsilon}(u) f_{\varepsilon}(y)$ is in $L^{1}\left(\mathbb{R}^{m+n}\right)$ and, by Fubini's Theorem,

$$
\begin{aligned}
\widetilde{H}_{\varepsilon}\left(f_{\varepsilon}\right)(x) & =\int\left(\int L_{\varepsilon}\left(\psi_{\varepsilon, y}\right)(x-u) f_{\varepsilon}(y) \mathrm{d} y\right) \varphi_{\varepsilon}(u) \mathrm{d} u \\
& =\left(\left\{\xi \mapsto \int L_{\varepsilon}\left(\psi_{\varepsilon, y}\right)(\xi) f_{\varepsilon}(y) \mathrm{d} y\right\} * \varphi_{\varepsilon}\right)(x) .
\end{aligned}
$$

An adaptation of the proof of Lemma 30 in [5] shows that, for all $\xi \in \mathbb{R}^{m}$, we have the following equality

$$
\begin{aligned}
\forall g \in \mathcal{D}\left(\mathbb{R}^{n}\right), \quad \int L_{\varepsilon}\left(\psi_{\varepsilon, y}\right)(\xi) g(y) \mathrm{d} y & =L_{\varepsilon}\left(\left\{v \mapsto \int \psi_{\varepsilon, y}(v) g(y) \mathrm{d} y\right\}\right)(\xi) \\
( & \left.=L_{\varepsilon}\left(\left\{v \mapsto \int \psi_{\varepsilon}(y-v) g(y) \mathrm{d} y\right\}\right)(\xi)\right) .
\end{aligned}
$$

(Indeed, the integrals under consideration in (31) are integrals of continuous functions on compact sets and can be considered as limits of Riemann sums in the spirit of [12], Lemma 4.1.3. The linearity and the continuity of $L_{\varepsilon}$ allows to exchange the order or the operations integral and $L_{\varepsilon}$.)

Then, a density argument shows that equality (31) holds for $g \in \mathcal{S}\left(\mathbb{R}^{n}\right)$. Thus

$$
\int L_{\varepsilon}\left(\psi_{\varepsilon, y}\right)(\xi) f_{\varepsilon}(y) \mathrm{d} y=L_{\varepsilon}\left(\left\{v \mapsto \int \psi_{\varepsilon}(y-v) f_{\varepsilon}(y) \mathrm{d} y\right\}\right)(\xi)=L_{\varepsilon}\left(\psi_{\varepsilon} * f_{\varepsilon}\right)(\xi)
$$

and $\widetilde{H}_{\varepsilon}\left(f_{\varepsilon}\right)(x)=\left(L_{\varepsilon}\left(\psi_{\varepsilon} * f_{\varepsilon}\right) * \varphi_{\varepsilon}\right)(x)$ as claimed.

We now complete the proof of Theorem 17 Set

$$
H_{L}=\left[\left(H_{\varepsilon}\right)_{\varepsilon}\right]_{\tau}=\left((x, y) \mapsto\left(\Psi_{\varepsilon, y} * \varphi_{\varepsilon}\right)(x)\right)_{\varepsilon}+\mathcal{N}_{\tau}\left(\mathbb{R}^{m+n}\right) .
$$


For all $\left(f_{\varepsilon}\right)_{\varepsilon}$ in $\mathcal{E}_{\mathcal{S}}^{\mathcal{L}_{a}}\left(\mathbb{R}^{n}\right)$, we have

$$
\widetilde{H}_{L}\left(\left[\left(f_{\varepsilon}\right)_{\varepsilon}\right]_{\mathcal{S}}\right)=\left[\left(\widetilde{H}_{\varepsilon}\left(f_{\varepsilon}\right)\right)_{\varepsilon}\right]_{\tau}
$$

by definition of the integral operator. We have to compare $\left(\widetilde{H}_{\varepsilon}\left(f_{\varepsilon}\right)\right)_{\varepsilon}$ and $\left(L_{\varepsilon}\left(f_{\varepsilon}\right)\right)_{\varepsilon}$. According to Lemma 22. we have for all $\varepsilon \in(0,1]$

$$
\begin{aligned}
\widetilde{H}_{\varepsilon}\left(f_{\varepsilon}\right)-L_{\varepsilon}\left(f_{\varepsilon}\right) & =\left(L_{\varepsilon}\left(\psi_{\varepsilon} * f_{\varepsilon}\right) * \varphi_{\varepsilon}\right)-L_{\varepsilon}\left(f_{\varepsilon}\right) \\
& =L_{\varepsilon}\left(\psi_{\varepsilon} * f_{\varepsilon}\right) * \varphi_{\varepsilon}-L_{\varepsilon}\left(f_{\varepsilon}\right) * \varphi_{\varepsilon}+L_{\varepsilon}\left(f_{\varepsilon}\right) * \varphi_{\varepsilon}-L_{\varepsilon}\left(f_{\varepsilon}\right) \\
& =L_{\varepsilon}\left(\psi_{\varepsilon} * f_{\varepsilon}-f_{\varepsilon}\right) * \varphi_{\varepsilon}+L_{\varepsilon}\left(f_{\varepsilon}\right) * \varphi_{\varepsilon}-L_{\varepsilon}\left(f_{\varepsilon}\right) .
\end{aligned}
$$

Remarking that $\left(f_{\varepsilon}\right)_{\varepsilon} \in \mathcal{E}_{\mathcal{S}}^{\mathcal{L}_{a}}\left(\mathbb{R}^{n}\right)$ and $\left(L_{\varepsilon}\left(f_{\varepsilon}\right)\right)_{\varepsilon} \in \mathcal{E}_{\tau}^{\mathcal{L}^{a+b c}}\left(\mathbb{R}^{m}\right) \subset \mathcal{E}_{\tau}^{\mathcal{L}_{1}}\left(\mathbb{R}^{m}\right)$, we get

$$
\left(L_{\varepsilon}\left(f_{\varepsilon}\right) * \varphi_{\varepsilon}-L_{\varepsilon}\left(f_{\varepsilon}\right)\right)_{\varepsilon} \in \mathcal{N}_{\tau}\left(\mathbb{R}^{m}\right) \text { and }\left(\psi_{\varepsilon} * f_{\varepsilon}-f_{\varepsilon}\right)_{\varepsilon} \in \mathcal{N}_{S}\left(\mathbb{R}^{m}\right)
$$

by Lemma 10] This last property gives

$$
\left(L_{\varepsilon}\left(\psi_{\varepsilon} * f_{\varepsilon}-f_{\varepsilon}\right)\right)_{\varepsilon} \in \mathcal{N}_{\tau}\left(\mathbb{R}^{m}\right) \text { and }\left(L_{\varepsilon}\left(\psi_{\varepsilon} * f_{\varepsilon}-f_{\varepsilon}\right) * \varphi_{\varepsilon}\right) \in \mathcal{N}_{\tau}\left(\mathbb{R}^{m}\right),
$$

since $\left(\eta_{\varepsilon} * \varphi_{\varepsilon}\right)_{\varepsilon} \in \mathcal{N}_{\tau}\left(\mathbb{R}^{m}\right)$ for all $\left(\eta_{\varepsilon}\right)_{\varepsilon} \in \mathcal{N}_{\tau}\left(\mathbb{R}^{m}\right)$. Finally

$$
\left[\left(\widetilde{H}_{\varepsilon}\left(f_{\varepsilon}\right)\right)_{\varepsilon}\right]_{\tau}=\left[\left(L_{\varepsilon}\left(f_{\varepsilon}\right)\right)_{\varepsilon}\right]_{\tau}=L\left(\left[\left(f_{\varepsilon}\right)_{\varepsilon}\right]_{\mathcal{S}}\right),
$$

this last equality by definition of the extension of a linear map.

\subsection{Proof of Proposition 20}

Assertion $(i)$.- For a fixed $\varepsilon \in(0,1], L_{\varepsilon}$ is obtained by composition of the continuous maps $\Lambda: \mathcal{S}\left(\mathbb{R}^{n}\right) \mapsto \mathcal{S}^{\prime}\left(\mathbb{R}^{m}\right)$ and

$$
\mathcal{S}^{\prime}\left(\mathbb{R}^{m}\right) \rightarrow \mathcal{O}_{M}\left(\mathbb{R}^{n}\right), \quad T \mapsto T_{k} * \varphi_{\varepsilon^{s}}
$$

Thus $L_{\varepsilon}$ is continuous. We have now to show that the net $\left(L_{\varepsilon}\right)_{\varepsilon} \in \mathcal{L}\left(\mathcal{S}\left(\mathbb{R}^{n}\right), \mathcal{O}_{M}\left(\mathbb{R}^{m}\right)\right)^{(0,1]}$ is strongly moderate. We have

$$
\begin{aligned}
\forall f \in \mathcal{S}\left(\mathbb{R}^{n}\right), \forall x \in \mathbb{R}^{m}, \forall \alpha \in \mathbb{N}^{m}, \quad \partial^{\alpha}\left(L_{\varepsilon}(f)\right)(x) & =\left(\Lambda(f) * \partial^{\alpha} \varphi_{\varepsilon^{s}}\right)(x) \\
& =\left\langle\Lambda(f),\left\{y \mapsto \partial^{\alpha} \varphi_{\varepsilon^{s}}(x-y)\right\}\right\rangle .
\end{aligned}
$$

The map

$$
\Theta: \mathcal{S}\left(\mathbb{R}^{n}\right) \times \mathcal{S}\left(\mathbb{R}^{m}\right), \quad(f, \varphi) \rightarrow\langle\Lambda(f), \varphi\rangle
$$

is a bilinear map, separately continuous since $\Lambda$ is continuous. As $\mathcal{S}\left(\mathbb{R}^{n}\right)$ and $\mathcal{S}\left(\mathbb{R}^{m}\right)$ are Fréchet spaces, $\Theta$ is globally continuous. There exist $C_{1}>0,\left(q_{1}, l_{1}, q_{2}, l_{2}\right) \in \mathbb{N}^{4}$, such that

$$
\forall(f, \varphi) \in \mathcal{S}\left(\mathbb{R}^{n}\right) \times \mathcal{S}\left(\mathbb{R}^{m}\right), \quad|\langle\Lambda(f), \varphi\rangle| \leq C_{1} \mu_{q_{1}, l_{1}}(f) \mu_{q_{2}, l_{2}}(\varphi) .
$$

In particular, for any $l \in \mathbb{N}$ and $\alpha \in \mathbb{N}^{m}$ with $|\alpha| \leq l$, we have

$$
\forall x \in \mathbb{R}^{m},\left|\left\langle\Lambda(f), \partial^{\alpha} \varphi_{\varepsilon}(x-\cdot)\right\rangle\right| \leq C_{1} \mu_{q_{1}, l_{1}}(f) \mu_{q_{2}, l_{2}}\left(\partial^{\alpha} \varphi_{\varepsilon^{s}}(x-\cdot)\right),
$$

with

$$
\begin{aligned}
\forall x \in \mathbb{R}^{m}, \mu_{q_{2}, l_{2}}\left(\partial^{\alpha} \varphi_{\varepsilon^{s}}(x-\cdot)\right) & =\sup _{\xi \in \mathbb{R}^{m},|\beta| \leq l_{2}}(1+|\xi|)^{q_{2}}\left|\partial^{\alpha+\beta} \varphi_{\varepsilon^{s}}(x-\xi)\right| \\
& =\sup _{\xi \in \mathbb{R}^{m},|\beta| \leq l_{2}}(1+|x-\xi|)^{q_{2}}\left|\partial^{\alpha+\beta} \varphi_{\varepsilon^{s}}(\xi)\right| \\
& \leq(1+|x|)^{q_{2}} \mu_{q_{2}, l_{2}+l}\left|\varphi_{\varepsilon^{s}}\right| .
\end{aligned}
$$


Using the definition of $\left(\varphi_{\varepsilon^{s}}\right)_{\varepsilon}$, we get $C_{2}>0$ such that $\mu_{q_{2}, l_{2}+l}\left|\varphi_{\varepsilon^{s}}\right| \leq C_{2} \varepsilon^{-s\left(m+l_{2}+l\right)}$, for $\varepsilon$ small enough. Thus, there exists $C>0$, such that, for $\varepsilon$ small enough,

$$
\forall x \in \mathbb{R}^{m}, \quad\left|\partial^{\alpha}\left(L_{\varepsilon}(f)\right)(x)\right|=\left|\left\langle\Lambda(f), \partial^{\alpha} \varphi_{\varepsilon}(x-\cdot)\right\rangle\right| \leq C(1+|x|)^{q_{2}} \mu_{q_{1}, l_{1}}(f) \varepsilon^{-s\left(m+l_{2}+l\right)} .
$$

Finally

$$
\mu_{-q_{2}, l}\left(L_{\varepsilon}(f)\right) \leq C \varepsilon^{-s\left(m+l_{2}+l\right)} \mu_{q_{1}, l_{1}}(f) .
$$

The sequence $r(\cdot)=\left\{l \mapsto s\left(m+l_{2}+l\right)\right\}$ satisfies $\lim _{l \rightarrow+\infty}(r(l) / l)=s<1$. Recalling that $l_{1}$ does not depend on $l$, we obtain our claim.

Assertion (ii).- We have the following:

Lemma 23 For all $u \in \mathcal{S}^{\prime}\left(\mathbb{R}^{m}\right),\left[\left(u * \varphi_{\varepsilon^{s}}\right)_{\varepsilon}\right]$ is equal to $u$ in the generalized distribution sense.

Proof. Take $u \in \mathcal{S}^{\prime}\left(\mathbb{R}^{m}\right)$. There exist $\alpha \in \mathbb{N}^{m}, q \in \mathbb{N}$, and $f: \mathbb{R}^{m} \rightarrow \mathbb{C}$ a continuous bounded function such that [21]

$$
u=\partial^{\alpha}\left(M^{q} f\right),
$$

where $M: \mathbb{R}^{m} \rightarrow \mathbb{C}$ is the function defined by $M(x)=1+|x|^{2}$. For any $g \in \mathcal{S}\left(\mathbb{R}^{m}\right)$, we have

$$
\left\langle u * \varphi_{\varepsilon^{s}}, g\right\rangle=\left\langle u, g * \check{\varphi}_{\varepsilon^{s}}\right\rangle=(-1)^{|\alpha|}\left\langle f, M^{q}\left(\partial^{\alpha} g\right) * \check{\varphi}_{\varepsilon^{s}}\right\rangle .
$$

On the other hand,

$$
\langle u, g\rangle=(-1)^{|\alpha|}\left\langle f, M^{q} \partial^{\alpha} g\right\rangle
$$

Thus

$$
\left\langle u * \varphi_{\varepsilon^{s}}, g\right\rangle-\langle u, g\rangle=\left\langle f, M^{q}\left(\left(\partial^{\alpha} g\right) * \check{\varphi}_{\varepsilon^{s}}-\partial^{\alpha} g\right)\right\rangle .
$$

A simplification of the proof of 10 shows that $\left(\left(\partial^{\alpha} g\right) * \check{\varphi}_{\varepsilon^{s}}-\partial^{\alpha} g\right)_{\varepsilon} \in \mathcal{N}_{\mathcal{S}}\left(\mathbb{R}^{d}\right)$. The same holds for $M^{q}\left(\left(\partial^{\alpha} g\right) * \check{\varphi}_{\varepsilon^{s}}-\partial^{\alpha} g\right)$. Thus, for all $p$ in $\mathbb{N}$,

$$
\left\langle u * \varphi_{\varepsilon^{s}}, g\right\rangle-\langle u, g\rangle=\mathrm{O}\left(\varepsilon^{p}\right) \text { as } \varepsilon \rightarrow 0 .
$$

This lemma implies that for all $f \in \mathcal{S}\left(\mathbb{R}^{n}\right),\left[\left(L_{\varepsilon}(f)\right)_{\varepsilon}\right]_{\tau}=\left[\left(\Lambda(f) * \varphi_{\varepsilon^{s}}\right)_{\varepsilon}\right]_{\tau}$ is equal to $\Lambda(f)$ in the generalized distribution sense. On the other hand, according to Theorem [17 $\left[\left(L_{\varepsilon}(f)\right)_{\varepsilon}\right]_{\mathcal{\tau}}=$ $\widetilde{H}_{L}(f)$ where $\widetilde{H}_{L}$ is the integral operator associated to the canonical extension of $\left(L_{\varepsilon}\right)_{\varepsilon}$. This ends the proof of Proposition 20.

\section{References}

[1] Antonevich A.B., Radyno Ya.V. On a general method for constructing algebras of generalized functions. Soviet Math. Dokl. 43(3):680-684, 1992.

[2] S. Bernard, J.-F. Colombeau, A. Delcroix. Generalized Integral Operators and Applications. Accepted in Math. Proc. Cambridge Philos. Soc.

[3] J.-F. Colombeau. New Generalized Functions and Multiplication of Distributions. NorthHolland (Amsterdam, Oxford, New-York), 1984.

[4] J.-F. Colombeau. Elementary introduction to New generalized Functions. North-Holland (Amsterdam, Oxford, New-York), 1985.

[5] A. Delcroix. Generalized Integral Operators and Schwartz Kernel Theorem. J. Math. Anal. Appl. 306(2):481-501, 2005.

[6] A. Delcroix. Regular nonlinear generalized functions and applications. Accepted in Bull. Cl. Sci. Math. Nat. Sci. Math, 2005. 
[7] A. Delcroix. Regular rapidly decreasing nonlinear generalized functions. Application to microlocal regularity. Preprint AOC, 2005.

[8] A. Delcroix, M. Hasler, S. Pilipović, V. Valmorin. Generalized function algebras as sequence space algebras. Proc. Amer. Math. Soc. 132:2031-2038, 2004.

[9] C. Garetro. Pseudo-differential Operators in Algebras of Generalized Functions and Global Hypoellipticity. Acta Appl. Math. 80(2):123-174, 2004.

[10] C. Garetto, T. Gramchev, M. Oberguggenberger. Pseudo-Differential operators and regularity theory. Preprint 8-2003, Preprint series of the department of Engineering Mathematics, Geometry and Computer Science, University of Innsbruck, http://techmath.uibk.ac.at/mathematik/publikationen

[11] M. Grosser, M. Kunzinger, M. Oberguggenberger, R. Steinbauer. Geometric Theory of Generalized Functions with Applications to General Relativity. Kluwer Academic Press, 2001.

[12] L. Hörmander. The analysis of Linear Partial Differential Operators I, distribution theory and Fourier Analysis. Grundlehren der mathematischen Wissenchaften 256. Springer Verlag (Berlin, Heidelberg, New York), 2nd edition, 1990.

[13] J.-A. MARTi. Non linear Algebraic analysis of delta shock wave to Burgers' equation. Pacific J. Math. 210(1):165-187, 2003.

[14] M. Nedelukov, S. Pilipović, D. ScarpalÉzos. The linear theory of Colombeau generalized functions. Pitman Research Notes in Mathematics Series 385. Longman, 1998

[15] M. Oberguggenberger. Multiplication of Distributions and Applications to Partial Differential Equations. Longman Scientific \& Technical, 1992.

[16] S. Pilipović, D. ScarpalÉzos. Colombeau generalized ultradistributions. Math. Proc. Cambridge Philos. Soc. 130:541-553, 2001.

[17] Ya.-V. Radyno, Ngo Fu Tkhan, S. Ramadan. The Fourier transform in an algebra of new generalized functions. Russian acad. Sci. Dokl. Math. 46(3):414-417, 1992

[18] D. ScarpalÉzos. Colombeau's generalized functions: Topological structures; Microlocal properties. A simplified point of view. Prépublication Mathématiques de Paris 7/CNRS, URA212, 1993.

[19] D. ScarpalÉzos. Colombeau's generalized functions: Topological structures; Microlocal properties. A simplified point of view. Part I. Bull. Cl. Sci. Math. Nat. Sci. Math. 25:89-114, 2000.

[20] D. Scarpalézos. Colombeau's generalized functions: Topological structures; Microlocal properties. A simplified point of view. Part II. Publ. Inst. Math. (Beograd) (N.S.) 76(90):111-125, 2004.

[21] L. Schwartz. Théorie des Distributions. Hermann (Paris), 3rd print, 1965.

[22] F. Treves. Topological vector spaces, distributions and kernels. Academic Press (New York, London), 1967.

[23] V. Valmorin. Schwartz kernel theorem in Colombeau algebras. Preprint AOC, 2005. 